\title{
La iconografía musical en el ámbito de las enseñanzas profesionales de música de conservatorio: una experiencia integradora
}

\author{
Carmen M. Zavala Arnal. Conservatorio Profesional de Música de Huesca \\ Recepción: 30 de julio de 2016 | Revisión: 6 de septiembre de 2016 | Aceptado: 26 octubre de 2016 \\ Correspondencia: Carmen Zavala | Email: czavala@unizar.es \\ Citar: Zavala, C. (2016). La iconografía musical en el ámbito de las enseñanzas profesionales de música de conservatorio: una \\ experiencia integradora. ReiDoCrea, 5, 255-262.
}

\begin{abstract}
Resumen: Este artículo se basa en una experiencia interdisciplinar realizada con alumnos de la asignatura de Historia de la Música en el marco de las enseñanzas profesionales de música de conservatorio, a partir de la iconografía musical representada en algunas obras plásticas conservadas. Se persigue que los alumnos enriquezcan su formación humanística, que adquieran algunos conocimientos básicos relacionados con la iconografía musical y su interés a la hora de ilustrar el hecho musical desde un punto de vista crítico y reflexivo, y que conozcan una parte del repertorio que integra el patrimonio histórico-artístico. Tras la realización de la experiencia, basada en la revista didáctica y en la ficha catalográfica como principales herramientas educativas, se ha podido constatar cómo ésta ha venido a mejorar la cultura visual y humanística de los alumnos, y a favorecer un aprendizaje más significativo e integrado.
\end{abstract}

Palabras clave: Educación musical | Iconografía

Music iconography in the field of music professional learning in conservatories: an integration experience

Abstract: This article is based on an interdisciplinary experience carried out with History of Music students within the framework of professional learning in conservatories, starting from iconography represented in some preserved plastic works. The ultimate aim is that the students enrich their humanistic training, acquire some knowledge about music iconography and its significance to help illustrate the musical act from a critical and reflective point of view, and become acquainted with a part of the repertoire, which makes up the historic and artistic heritage. After this experience, based on didactics journals and card catalogues as main teaching tools, it has been confirmed how it has improved the students' visual and humanistic culture and how it has fostered a more significant and integrated learning.

Key words: Music education | Iconography

\section{Introducción}

La iconografía musical es una rama de la musicología que estudia las representaciones musicales en las artes visuales (Mayer-Brown, 1980). Desde ámbitos institucionales y universitarios se ha favorecido en las últimos décadas la creación de grupos de investigación sobre iconografía musical, dedicados principalmente a la catalogación de obras de arte con elementos musicales (Bethencourt, Carvajal, Palacios, 2012). Respecto a su aplicación en el contexto de la enseñanza musical, se han realizado algunos estudios, aunque referidos a la producción y recreación de imágenes musicales por parte de los alumnos a través de trabajos de artes plásticas y manualidades (Cartas, 2006; Sarfson, 2015). En el ámbito universitario, el uso de la iconografía musical en el aprendizaje de la música, ha sido recientemente estudiado en una tesis doctoral (Zavala, 2015), en la que se realiza una aplicación didáctica a partir de obras pictóricas, en el marco de las enseñanzas musicales universitarias destinadas a la formación de futuros profesionales de la enseñanza. En ella, se insiste en la importancia del conocimiento de la imagen musical a través del estudio histórico-artístico de la obra y del tema artístico-iconográfico representado. Por otro lado, el uso de la iconografía musical en el ámbito educativo favorece el conocimiento del patrimonio histórico-artístico (Zavala, Ramón, 2016).

En el contexto de las enseñanzas profesionales de música, impartidas en los conservatorios profesionales, la iconografía musical se presenta como un interesante recurso que viene a enriquecer los conocimientos de carácter histórico, estético y organológico que los alumnos han de adquirir en esta etapa de su formación. Así, la 
asignatura de Historia de la Música, presente en el quinto y sexto curso de enseñanzas profesionales según el currículo actual vigente, resulta el marco idóneo para realizar una experiencia desde la iconografía musical.

La Historia de la Música en las enseñanzas profesionales de música debe introducir a los alumnos, no sólo al estudio de los diferentes estilos y modos de creación musical, sino que ha de tener también una orientación práctica y experimental. Además, han de entender la evolución de la música a lo largo del tiempo a través de su estrecha conexión con el resto de las artes a través del uso de fuentes de información histórica. En este sentido, la iconografía musical se muestra como una herramienta útil que, a partir de determinadas pautas que orienten en la adquisición de evidencias musicológicas (Winternitz, 1979, Roubina, 2010), puede contribuir a la adquisición de algunos contenidos específicos de la asignatura. Por otro lado, el conocimiento de la obra de arte, que es el soporte en el que se reflejan las imágenes musicales, brinda a los alumnos otra vía a través de la cuál pueden valorar el patrimonio histórico-artístico.

Con respecto al objetivo principal de esta investigación, se ha pretendido realizar una aplicación didáctica sobre iconografía musical en el ámbito de las enseñanzas profesionales de música de conservatorio a través de un material iconográfico-musical seleccionado y adaptado, que muestre cómo esta viene a favorecer y enriquecer la formación musical y humanística de los alumnos.

\section{Método}

\section{Participantes}

Esta aplicación basada en la iconografía musical se ha realizado en el Conservatorio Profesional de Música de Huesca, en la asignatura Historia de la Música, impartida en el quinto y sexto curso de Enseñanza Profesionales. La suma de los grupos lo configuran un total de 31 alumnos y alumnas, de edades comprendidas mayoritariamente entre los 15 y los 18 años.

\section{Instrumentos}

Los principales instrumentos didácticos utilizados han sido la revista, la ficha catalográfica, y el cuestionario para la medición de datos.

El uso de la revista como herramienta didáctica favorece la comunicación entre los miembros de la comunidad educativa y mejora los aspectos formativos y sociales del proceso de enseñanza-aprendizaje (Fernández, Rodríguez, 2001). Se trata además de un instrumento versátil que permite la difusión de sus contenidos a través de diversas vías: en formato papel, distribuidas en el centro educativo; a través del correo electrónico, enviado a alumnos, profesores, padres y madres, etc.; y a través de la página web del centro educativo, para cualquier usuario interesado en los temas que se abordan.

Por otra parte, se utiliza un modelo de ficha para la catalogación de obras artísticas con iconografía musical que incluye campos relacionados con aspectos formales, con contenidos musicales, y con el estudio de los temas artísticos e iconográficos y sus fuentes (Bordas, 2001; Piquer, Rodríguez, 2012), adaptada al contexto de la asignatura. La utilidad de las fichas catalográficas en el campo de la enseñanza viene dada por su propia estructura, que las convierte en una herramienta útil a la hora de transmitir contenidos de manera sintética. 
La metodología didáctica de esta experiencia se basa principalmente en una metodología experimental (León, Montero, 1993), en la que se diseñan procedimientos conducentes a obtener datos cuantificables a partir de actividades de toma de muestras, con el fin de desencadenar un proceso de investigación-acción que mejore el proceso de enseñanza y aprendizaje de un grupo determinado. A través del método cuantitativo se produce la creación de unos cuestionarios originales, de carácter anónimo, que se relacionan con la obtención de unos resultados, y con la emisión de unas conclusiones finales en relación con la confirmación o falsación de las hipótesis. De esta forma, el paradigma cuantitativo nos brinda una determinada información desde una perspectiva realista y estable en un escenario determinado, el aula de conservatorio, a través de las mediciones cuantitativas que proporcionan los cuestionarios realizados dos grupos de alumnos en la asignatura Historia de la Música de Enseñanza Profesionales Música.

Por otra parte, se tienen en cuenta los postulados de la investigación-acción que se plantea, en aras enriquecer el currículum formativo de los alumnos de historia de la música, así como sus procesos de aprendizaje. En este sentido, se considera el concepto de "proceso" como una serie de actuaciones o actividades a través de las cuales determinados inputs, esto es, recursos, alumnos sin formar, planes, etcétera, que añaden valor y dan lugar a outputs, entendidos como resultados, alumnos formados, etcétera (Sarfson, 2015).

\section{Procedimiento}

Para la realización de esta experiencia se parte de una sección realizada en los tres números, correspondientes a los tres trimestres lectivos del curso 2015/2016, de la Revista de Viento y Percusión del Conservatorio de Música de Huesca. En la sección titulada "Adivina dónde están...", se propone a los alumnos del centro que averigüen en qué espacios de la ciudad se encuentran conservadas tres obras artísticas, realizadas entre 1285 y 1500 , cuyos datos se obtienen a partir de bibliografía seleccionada, en las que se representan instrumentos de viento. En el siguiente número de la revista se publica la solución, detallando aspectos relevantes de la imagen musical. A través de esta actividad, los alumnos pueden observar ciertos aspectos organológicos de los instrumentos de viento a través de las obras artísticas, y reflexionar sobre su significado y simbología. Además, les permite el conocimiento del patrimonio artístico local, instándoles a visitar museos y otros espacios de la ciudad, y fomenta la realización de actividades de tipo interdisciplinar en las que se relaciona la materia musical con la plástica.

Posteriormente, se realiza una actividad instruccional basada en la transmisión de contenidos sobre iconografía musical a partir de la adaptación de la información de las obras artísticas, de las que ya tienen referencia a través de la citada revista, a su nivel curricular. Con anterioridad, los alumnos ya habían asimilado los contenidos relacionados con la música medieval y renacentista y su contexto, bien en el primer trimestre del curso presente (alumnos de $5^{\circ}$ de Historia de la Música), bien en el curso anterior (alumnos de $6^{\circ}$ de la misma asignatura), conocimientos que, por otro lado, son necesarios para la realización de la actividad sobre iconografía musical. Esta actividad de aprendizaje específico viene a aportar, por tanto, la adquisición por parte de los alumnos de nuevos conocimientos en el campo de la iconografía musical, a través de su definición y comparación, y de una interpretación crítica; y a través de la ampliación de la información sobre las obras artísticas seleccionadas, utilizando como material primario las fichas catalográficas adaptadas. Como paso previo, los alumnos realizan un cuestionario inicial, a través del cual se obtiene información sobre la naturaleza de 
los dos grupos de alumnos, así como sobre su grado de interés y los conocimientos previos que tienen sobre el asunto principal y secundarios de la aplicación.

Finalmente, tras la transmisión de contenidos, se realiza un cuestionario final que nos permite averiguar si se cumplen los objetivos, y si las preguntas provocan el tipo de respuesta esperada tras la realización de las actividades de aprendizaje sobre iconografía musical.

\section{Análisis de datos}

Para la obtención de datos acerca de los resultados de esta experiencia sobre iconografía musical se realiza un cuestionario final que nos permite averiguar si se han cumplido las expectativas didácticas.

Tal y como hemos recogido en al apartado de Instrumentos, se ha partido del método cuantitativo tomando como herramienta específica para el análisis de datos el cuestionario, que nos permite evaluar una realidad determinada; y para contabilizar los registros que nos proporciona, se ha utilizado la medición nominal, que expresa en porcentajes la presencia de variables dentro de cada categoría (Ferrando, 1992). Las fases que se han seguido para la elaboración de los cuestionario son las siguientes:

- Determinación del objetivo e hipótesis: la formulación de hipótesis previas es la base de los cuestionarios, por lo que se precisa estructurar desde el principio el objetivo principal, que ya hemos detallado,

- Elaboración de cuestionarios de carácter previo, o cuestionario inicial: en el que se incluyen preguntas cerradas, en las que el alumno selecciona de una serie de categorías la respuesta que más se aproxima a su opinión, que pueden ser dicotómicas y/o de elección múltiple; y preguntas abiertas, en las que el alumno responde con sus propias palabras.

- Elaboración de un cuestionario que contiene el mismo tipo de preguntas que el inicial Es importante subrayar que este último cuestionario, sin privarle de su naturaleza cuantitativa, se refiere fundamentalmente a la consideración de los alumnos de Historia de la Música acerca de los contenidos aprendidos sobre iconografía musical, y no a una comprobación estricta de los conocimientos alcanzados.

Finalmente, a través de los datos proporcionados por los cuestionarios, obtenemos unos porcentajes a modo de resultados, que nos suministran el conocimiento de un fenómeno real, y con los que realizamos los gráficos.

\section{Resultados}

Tras la realización de las actividades que conforman la experiencia sobre iconografía musical basada en un repertorio de obras plásticas, la mayor parte de los alumnos ha mostrado una gran motivación hacia la ampliación de conocimientos musicales y artísticos, manifestando un alto grado de interés por la historia de la música (bastante: $61 \%$, mucho: $32 \%$ ) y la historia del arte (bastante: $58 \%$, mucho: $26 \%$ ) como materias: 
Gráfico 1. Interés por la Historia de la Música

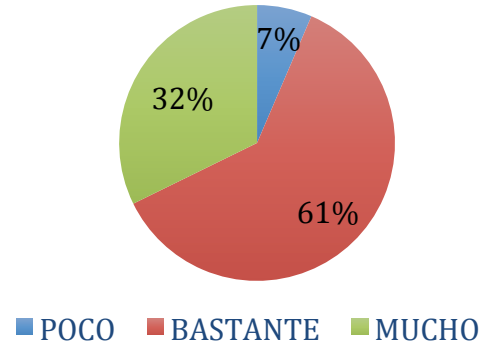

Gráfico 2. Interés por la Historia del Arte.

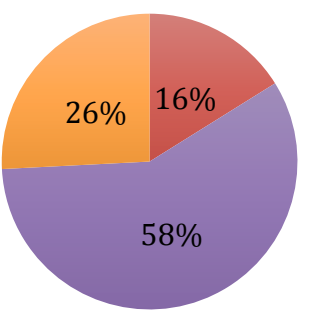

- POCO BASTANTE MUCHO

Además, han mostrado su alto grado de valoración del patrimonio artístico local $(42 \%$ bastante, $26 \%$ mucho, $22 \%$ muchísimo), tanto como fuente para la adquisición de conocimientos como para enriquecer su formación como músicos. También valoran positivamente el visionado directo de las obras de arte en su lugar de conservación, (29\% bastante $42 \%$ mucho, $23 \%$ muchísimo):

Gráfico 3. Importancia de conocer el patrimonio histórico-artístico local

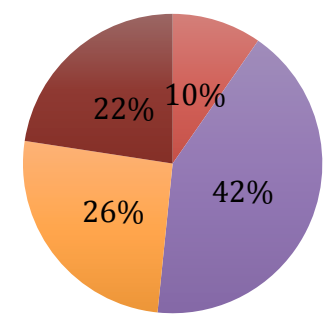

- POCO BASTANTE MUCHO घUCHÍSIMO 
Gráfico 4. ¿Valoras ver la obra de arte en su lugar de conservación?

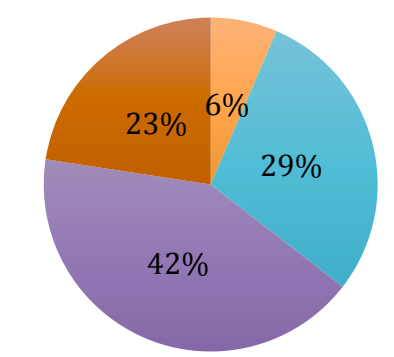

- POCO BASTANTE MUCHO - MUCHÍSIMO

La mayoría de los alumnos afirma haber asimilado el concepto de iconografía musical $(77 \%)$ :

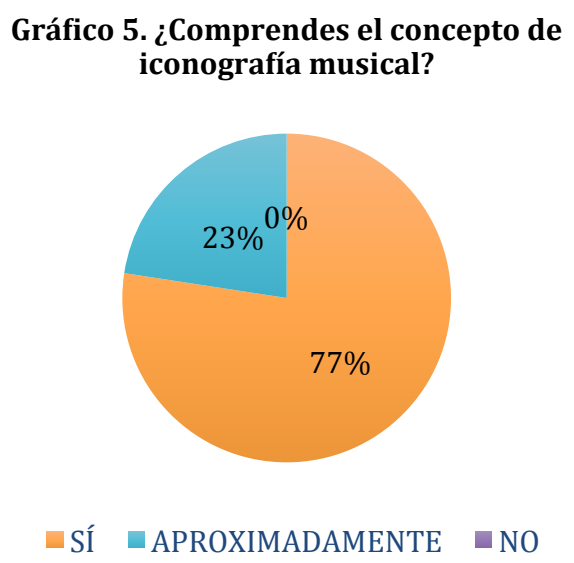

Por otro lado, manifiestan en su mayoría que ha mejorado su motivación hacia el conocimiento de historia del arte y de la música, y sus interrelaciones $(64 \%$ bastante, $26 \%$ mucho). La opinión generalizada es que han ampliado su formación en los aspectos desarrollados, por lo que podemos concluir que se han alcanzado los objetivos propuestos.

Gráfico 6. ¿Has aprendido cuestiones nuevas sobre la relación música-arte?

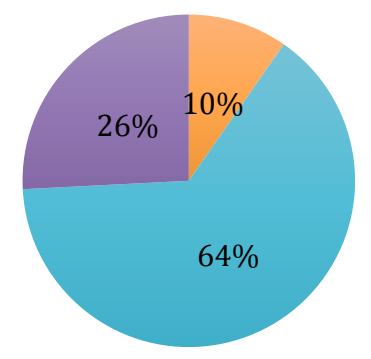

$\square$ POCO BASTANTE $\square$ MUCHO 


\section{Discusión}

La experiencia sobre iconografía musical que aquí se muestra ha pretendido contribuir al conocimiento de su uso como recurso didáctico en el contexto de la enseñanza musical a través de la realización de actividades de aprendizaje dentro y fuera del aula, basadas en una metodología que se fundamenta en el uso didáctico de la ficha catalográfica y el visionado de la obra de arte, y que aborda con una mirada global el campo de la música y las artes plásticas, así como sus ámbitos de interacción.

Por otro lado, se han alcanzado los objetivos propuestos respecto a los alumnos de la asignatura de Historia de la Música, impartida en las enseñanzas profesionales de música de conservatorio, ya que se ha constatado que esta experiencia ha sido muy útil para su formación, y les ha permitido integrar disciplinas afines, en aras de enriquecer tanto su aprendizaje musical como su cultura humanística y visual.

En relación a otros estudios realizados, los alumnos de conservatorio de quinto y sexto curso de enseñanzas profesionales han mostrado un mayor grado de compresión e interés por la iconografía musical, así como por el conocimiento del patrimonio histórico-artístico, respecto a los alumnos universitarios de Grado en Magisterio en Educación Primaria (Zavala, 2015), a pesar de que estos últimos tienen mayor madurez intelectual debido a su edad. Esto puede encontrar su explicación en que los estudiantes de magisterio adolecen en su mayoría de una formación musical especializada previa. Por otro lado, los alumnos de conservatorio poseen en muchos casos un acervo cultural más rico, fundamentado en el conocimiento, a lo largo de los años, de un repertorio histórico-musical que propicia su interés hacia las artes en general.

Podemos concluir que, a través de lo aquí expuesto, se ha tratado de sentar algunas bases para profundizar en el futuro en aspectos relacionados con la iconografía musical y su aplicación en el ámbito educativo, en especial, por su carácter integrador de conocimientos musicales, histórico-artísticos y humanísticos.

\section{Referencias}

Bethencourt, F., Carvajal, A. y Palacios, M. (2012). Aspectos científicos y modelos de investigación en España. El proyecto UCM. En C. Bordas Ibáñez e I. Rodríguez López (Eds.). Imagen es Música. Recursos para la catalogación y estudio de fuentes de Iconografía Musical en España y Portugal (22-45). Pozuelo de Alarcón (Madrid): Asociación Española de Documentación Musical.

Bordas Ibáñez, C. (2001). Música y artes plásticas. Una presentación y otra mirada más al Pórtico de la Gloria. Campos Interdisciplinares de la Musicología, Revista de Musicología, II. Madrid: Sociedad Española de Musicología,1227-1241.

Cartas Martín, I. (2006). Iconografía musical infantil. Icono, 14(7), 1-24.

Fernández Garcia, T. y Rodríguez Martín, V. (2001). Medios de comunicación y educación. En T. Fernández García y A. García Rico (Eds.). Medios de comunicación, sociedad y educación (121-142). Cuenca: Editorial de la Universidad de Castilla-La Mancha.

Ferrando, M. (1992). La Encuesta. El análisis de la realidad social: métodos y técnicas de investigación. Madrid: Alianza.

León García, O. y Montero García-Celay, I. (1993). Diseño de investigaciones. Introducción a la lógica de la investigación en psicología y educación. Madrid: McGraw-Hill, Interamericana de España.

Mayer Brown, H. (1980). Iconography of music. The New Grove Dictionary of Music and Musicians. Londres: Macmillan.

Piquer Sanclemente, R. y Rodríguez López, I. (2012). Criterios de catalogación y propuestas de estudio. Instrumentos para la catalogación de Iconografía Musical, En C. Bordas Ibáñez e I. Rodríguez López (Eds.). Imagen es Música. Recursos para la catalogación y estudio de fuentes de Iconografia Musical en España y Portugal (109-195). Pozuelo de Alarcón (Madrid): Asociación Española de Documentación Musical. 
Roubina, E. (2010). ¿Ver para creer?: una aproximación metodológica al estudio de la iconografía musical novohispana. Pesquisa em música: novas conquistas e novos rumos. I Simpósio Brasileiro de Pós-graduandos em Música da Unirio (63-83). Río de Janeiro (Brasil): Unirio.

Sarfson Gleizer, S. (2015). Iconografía musical y su didáctica: entre la mímesis y el símbolo en el contexto de la formación de maestros. REDU. Revista de Docencia Universitaria, 13(2), 307-321.

Zavala Arnal, CM. (2015). Iconografía musical en la pintura gótica aragonesa (Ca. 1300-1500): catálogo y estudio histórico-artístico. Una aplicación en la enseñanza musical universitaria (Tesis Doctoral inédita). Zaragoza: Universidad de Zaragoza.

Zavala Arnal, CM. y Ramón Salinas, J. (2016). El museo como espacio interdisciplinar. Una reflexión acerca de la contribución de la iconografía musical en la didáctica del patrimonio en la educación secundaria. En Actas del I Congreso Internacional 'Creando ciudadanos, construyendo identidades' (en prensa). Murcia: Universidad de Murcia.

Winternitz, E. (1979). Musical Instruments and Their Symbolism in Western Art. Londres: Yale University Press. 